\title{
The Effect of the Axisymmetric and Non-Axisymmetric Ambient Field on the Magnetic Field of Planets
}

\author{
Alhashmi Ali Darah1, Omran Moftah Trifis² \\ ${ }^{1}$ Mathematics Department, Faculty of Education, University of Alasmariya, Zliten, Libya \\ ${ }^{2}$ Physics Department, Faculty of Education, University of Alasmariya, Zliten, Libya \\ Email: a.a.adrah@gmail.com
}

How to cite this paper: Darah, A.A. and Trifis, O.M. (2021) The Effect of the Axisymmetric and Non-Axisymmetric Ambient Field on the Magnetic Field of Planets. Open Access Library Journal, 8: e7647.

https://doi.org/10.4236/oalib.1107647

Received: June 15, 2021

Accepted: August 16, 2021

Published: August 19, 2021

Copyright $\odot 2021$ by author(s) and Open Access Library Inc.

This work is licensed under the Creative

Commons Attribution International

License (CC BY 4.0).

http://creativecommons.org/licenses/by/4.0/ (c) (i) Open Access

\begin{abstract}
At least a minority of planets, moons and other bodies exist within significant external astrophysical fields. The ambient field problem is more relevant to these bodies than the classical dynamo problem, but remains relatively little studied. This paper will concern with the effect of axisymmetric and non-axisymmetric ambient field on a spherical, axisymmetric dynamo model, through nonlinear calculations with $\alpha$-quenching feedback. Ambient fields of varying strengths are imposed in the model. The effect of these fields on the generation process is considered, with particular focus on the equatorial symmetry and the time dependence of the solutions.
\end{abstract}

\section{Subject Areas}

Number Theory, Numerical Mathematics

\section{Keywords}

Mean-Field Dynamo, Axisymmetric, Nonlinear, Ambient Field

\section{Introduction}

In the presence of an ambient field, however, the critical nature of the field generation process is relaxed (Darah et al. 2005) [1]. Motions can distort and amplify the ambient field for all amplitudes of flow. For motions with appropriate geometries for regeneration, an internal 'dynamo-like' field of appreciable strength can be generated, even for relatively weak flows.

It meant, by external, that the contributions from the draped Interplanetary Magnetic Field IMF and from currents flowing on the planet's ionosphere either by in- 
duction from solar wind interactions or from ionospheric winds interacting with the crustal field. The last would be only possible over the regions where the crustal magnetic field is strong enough to interact with charged particle motion (Ferguson et al. 2005) [2].

Magnetic fields protect planets and atmospheres from solar particles. The particles from the Sun are charged, which means they respond to the magnetic field and move around it. Magnetic fields are, generally, generated by the movement of magnetic material located inside the planet, usually at the core. The Earth magnetic field, for example, is generated by liquid metal at the core and Earth's rapid rotation of 24 hours generates enough movement of the liquid to stimulate a magnetic field. The other planets in our solar system, except for Venus and Mars, all have magnetic fields or traces of magnetism that differ from Earth in various ways. So, how are other planetary magnetic fields generated?

Proximity to the Sun (seasonal cycle) causes an increase in solar wind pressure and therefore an overall increase in the magnetic field $\boldsymbol{B}$. Additionally, by using the magnetic field as a solar wind pressure proxy (Crider et al., 2003) [3], it could infer that solar events, fluctuations of solar wind pressure, will cause aperiodic variations in the external field.

Some structures in the external field result from interaction with crustal fields (Acufia et al., 2001 and Brain et al., 2003) [4] [5].

Most of the Magnetic Field observed at the Earth's surface has an internal origin. It is mainly produced by the dynamo action of turbulent flows in the fluid metallic outer core of the planet, while little is due to external magnetic fields located in the ionosphere and the magnetosphere (Qamili et al., 2013) [6]. It is the presence of the global magnetic field that, through the magnetosphere, protects the Earth, together with its biosphere, from the solar wind deflecting most of its charged particles (Occhipinti et al., 2014) [7].

The Martian magnetic field environment is a result of interactions among the internal crustal field and external fields, including the interplanetary magnetic field (IMF) and fields generated by ionospheric currents. Mars magnetic fields have been measured by fluxgate magnetometers on the Mars Global Surveyor (MGS) and Mars Atmosphere and Volatile Evolution Mission (MAVEN) missions.

The global external magnetic fields on Mars have been investigated using the MGS mapping orbit data set from 2000 to 2006, (Natalia et al., 2010) [8]. Moreover, it is apparent from the observations that there are significant external contributions to the magnetic field at Mapping Phase Orbit altitudes even on the nightside of Mars (Ferguson et al. 2005) [2].

The magnetic field of Mercury is critical to obtaining currents and the distribution of electric fields and flows within the magnetosphere (Glassmeier 1997) [9].

The external field arising from the interaction of the magnetosphere with the solar wind is more prominent near Mercury than for any other magnetized pla- 
net in the solar system (Brain et al. 2010) [10].

In contrast to planets like the Earth, Jupiter or Saturn, Venus does not have an intrinsic magnetic field and thus its atmosphere is not protected against the incoming solar wind. However, the strong solar radiation ionizes the upper atmosphere creating an ionosphere which in turn interacts with the solar wind ions and the magnetic field carried by the solar wind. This interaction leads to the formation of a magnetosphere: the solar wind magnetic field lines are "folded" around Venus, so the plasma flow is directed around the planet. Depending on the balance between internal pressure (exerted by ionospheric plasma) and external pressure (exerted by solar wind plasma), the positions of ionopause and magnetopause vary in altitude.

In a few cases (notably the Galilean satellites Europa and Callisto) there are induced fields arising from the time variation of an external field. These are also telling us something important about the body. The observed fields of Europa and Callisto are consistent with an externally induced induction field, and the most likely conductor is salty water (Stevenson 2000) [11].

\section{Model}

Most of studies of generating the magnetic field in celestial bodies are focused around the magnetic field generated internally in celestial bodies, whereas, some of the bodies are exposed to an external magnetic field, in one way or another, that is generated from other bodies independent of the affected body. So this paper will be concerned with external; axisymmetric and non-axisymmetric magnetic fields.

The study will consider the induction equation for both types of wavenumber in our spherical shell model; first, with an axisymmetric ambient field than with a non-axisymmetric ambient field. The system consists of an electrically conducting fluid contained in a spherical shell, and the region interior to the fluid is finitely conducting, and is of the same conductivity as the fluid itself.

$\boldsymbol{U}$ is the fluid flow. $\boldsymbol{B}^{i}$ and $\boldsymbol{B}^{o}$ are inner and outer core magnetic fields respectively, and $\boldsymbol{B}_{0}$ is an ambient magnetic field for the axisymmetric equations. And $\boldsymbol{b}^{i}$ and $\boldsymbol{b}^{o}$ are inner and outer core magnetic fields respectively, and $\boldsymbol{b}_{1}$ is an ambient magnetic field for the non-axisymmetric equations.

$$
\frac{\partial \boldsymbol{B}^{i}}{\partial t}=\nabla^{2} \boldsymbol{B}^{i}
$$

for the inner core field $\boldsymbol{B}^{i}$,

$$
\frac{\partial \boldsymbol{B}^{o}}{\partial t}=\nabla^{2} \boldsymbol{B}^{o}+\nabla \times\left[\boldsymbol{U} \times\left(\boldsymbol{B}^{o}+\boldsymbol{B}_{0}\right)+\alpha\left(\boldsymbol{B}^{o}+\boldsymbol{B}_{0}\right)\right]
$$

for the outer core field $\boldsymbol{B}^{o}$, and

$$
\nabla^{2} \boldsymbol{B}^{e}=0
$$

for the exterior field $\boldsymbol{B}^{e}$. The magnetic field is everywhere solenoidal:

$$
\nabla \cdot \boldsymbol{B}=0
$$


And the non-axisymmetric equations are

$$
\begin{gathered}
\frac{\partial \boldsymbol{b}^{i}}{\partial t}=\nabla^{2} \boldsymbol{b}^{i} \\
\nabla \cdot \boldsymbol{b}^{i}=0
\end{gathered}
$$

for inner core magnetic field $\boldsymbol{b}^{i}$; and

$$
\begin{gathered}
\frac{\partial \boldsymbol{b}^{o}}{\partial t}=\nabla \times\left[\boldsymbol{U} \times\left(\boldsymbol{b}^{o}+\boldsymbol{b}_{1}\right)+\alpha\left(\boldsymbol{b}^{o}+\boldsymbol{b}_{1}\right)\right]+\nabla^{2} \boldsymbol{b}^{o} \\
\nabla \cdot \boldsymbol{b}^{o}=0
\end{gathered}
$$

for inner core magnetic field $b^{i}$ and for outer core magnetic field $b^{o}$, e.g., Barenghi (2003) [12] and Hollerbach and Jones (1993) [13]. The flow $\boldsymbol{U}$ and $\alpha$-effect are axisymmetric, we assumed, as e.g., Brandenburg et al. (1989) [14], a functional form for $\alpha$ given by

$$
\alpha=\frac{\alpha_{0} \cos \theta}{1+\boldsymbol{b}^{2}}
$$

where $\alpha_{0}$ is a constant. The equations are non-dimensionalised using the lengthscale of the shell, $\mathcal{L}=\left(r_{o}-r_{i}\right)$, where $r_{o}$ and $r_{i}$ are the outer and inner core radii, and the magnetic diffusion time-scale, $\mathcal{T}=\mathcal{L}^{2} / \eta$, where $\eta$ is the magnetic diffusivity. This leaves the mean-field equations governed by the non-dimensional parameters

$$
R_{\omega}=\frac{\omega_{0} \mathcal{L}^{2}}{\eta}, \quad R_{\alpha}=\frac{\alpha_{0} \mathcal{L}}{\eta}
$$

In the following, we will fix both $R_{\omega}$ and $R_{\alpha}$ and treat $\boldsymbol{B}_{0}$ and $\boldsymbol{b}_{1}$ as our control parameter. The radius ratio $r_{i} / r_{o}=1 / 3$ is adopted. (This value is approximately that applicable to the Earth, here considered a model for other terrestrial planets). The boundary conditions on the magnetic field are derived from matching the outer and inner fields at $r=r_{i}$ and matching the outer field to the field in an insulator at $r=r_{o}$.

\section{Results}

The investigation will be concerned with both; the axisymmetric and non-axisymmetric solutions. One of exited solutions was at $R_{\omega}=R_{\alpha}=50$, as (Darah and Sarson 2005) [1], so the study will be related to the same values of $R_{\omega}$ and $R_{\alpha}$. In the paper the solutions will be noted by $\mathrm{O}$ for oscillatory and $\mathrm{S}$ for stationary behaviours respectively, and D for dipole and Q for quadruple solutions respectively.

\subsection{No Ambient Field $B_{0}=b_{1}=0$}

The solutions with no ambient field have an OD solution with average 11.7095 for the axisymmetric and a quasi OQ solution with average 0.76516 for the nonaxisymmetric solution (Figure 1). 


\subsection{With Axisymmetric Ambient Field $B_{0} \neq 0, b_{1}=0$}

\subsubsection{The Axisymmetric Solution}

The solution has the same behaviour, OD, as the previouse method; with no ambient field, and increases in the value for $\boldsymbol{B}_{0}=0.0-0.44$. At the range of $0.45<\boldsymbol{B}_{0}<0.49$ it is wavering between SD and OD with an average around of 9.5. After that, it becomes SD up to $\boldsymbol{B}_{0}=0.745$, where it becomes wavering again as before. At $\boldsymbol{B}_{0}=0.747$ it jumps to a higher amount, about 27.533892 with SD solution, and increases rapidly (Figure 2).

\subsubsection{The Non-Axisymmetric Solution}

The solution has the same behavior; QO, as the previouse method; with no ambient field, and it increases with increasing $\boldsymbol{B}_{0}$ up to $\boldsymbol{B}_{0}=0.41$. At $\boldsymbol{B}_{0}=0.42$ it moved to OD before it becomes wavering between $\mathrm{SD}$ and $\mathrm{OD}$ at the range of $0.45<\boldsymbol{B}_{0}<0.49$. For $0.50<\boldsymbol{B}_{0}<0.745$ it has a SQ solution then QO for $\boldsymbol{B}_{0}=0.746$. Finally it disappeared at $\boldsymbol{B}_{0}=0.747$ (Figure 3).

\subsection{With a Non-Axisymmetric Ambient Field $B_{0}=0, b_{1} \neq 0$}

\subsubsection{The Axisymmetric Solution}

Aucully, there are two solutions, the first is extunsion of the base solution OD,

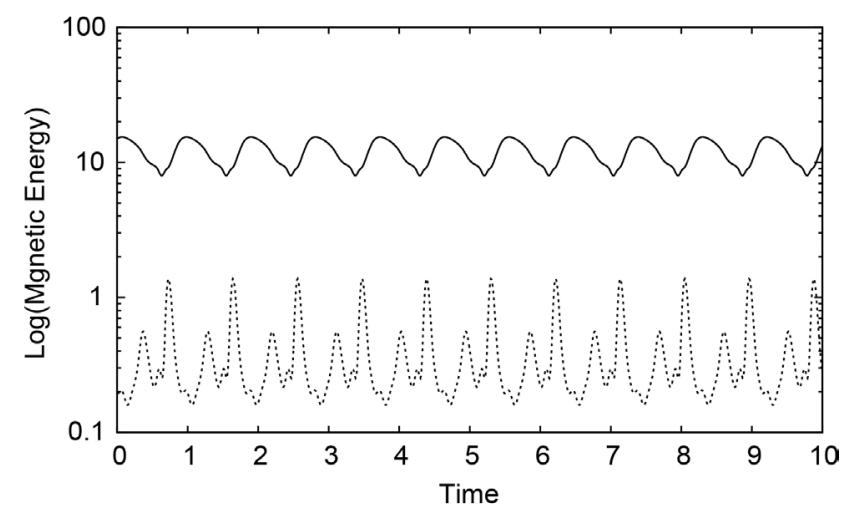

Figure 1. The OD axisymmetric magnetic energy (bold line) and OQ non-axisymmetric magnetic energy (dashed line) solutions for $\boldsymbol{B}_{0}=\boldsymbol{b}_{1}=0$.

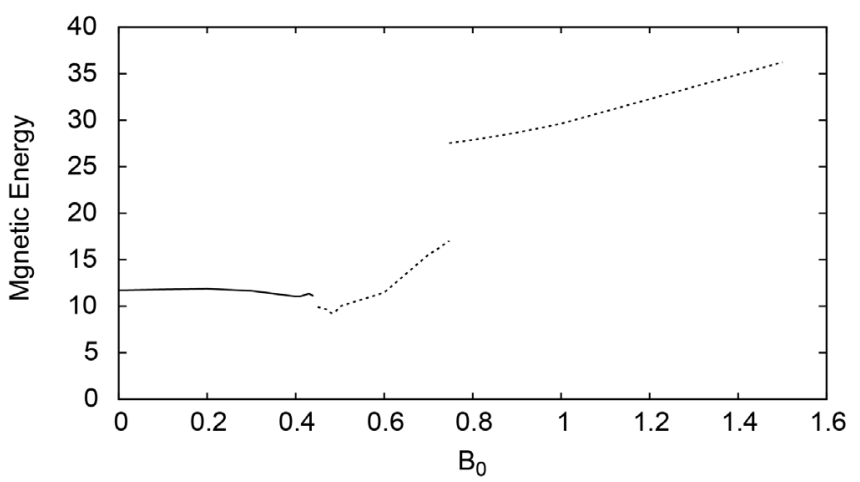

Figure 2. The OD magnetic energy for the axisymmetric (soled line) and SD (dashed line) solutions for the system with $\boldsymbol{B}_{0} \neq 0, \boldsymbol{b}_{1}=0$. 
with no ambient field, this solution decreases with increasing of $\boldsymbol{b}_{1}$ up to $\boldsymbol{b}_{1}=4.1$ where the solution turens to the second solution SD with value 12.364559 and continuous to decrease by increasing of $\boldsymbol{b}_{1}$, this solution back down up to $\boldsymbol{b}_{1}=0$, (Figure 4).

\subsubsection{The Non-Axisymmetric Solution}

The solution has the same behavior $\mathrm{OQ}$, as for $\boldsymbol{b}_{1}=0.0$, and continuous increasing as $\boldsymbol{b}_{1}$ increases up to $\boldsymbol{b}_{1}=4.0$. At, $\boldsymbol{b}_{1}=4.1$ it turns to SQ with value 10.665227 and increases rapidly by increasing of $\boldsymbol{b}_{1}$, this stationary solution refluxes back to vanish at $\boldsymbol{b}_{1}=0 \quad$ (Figure 5).

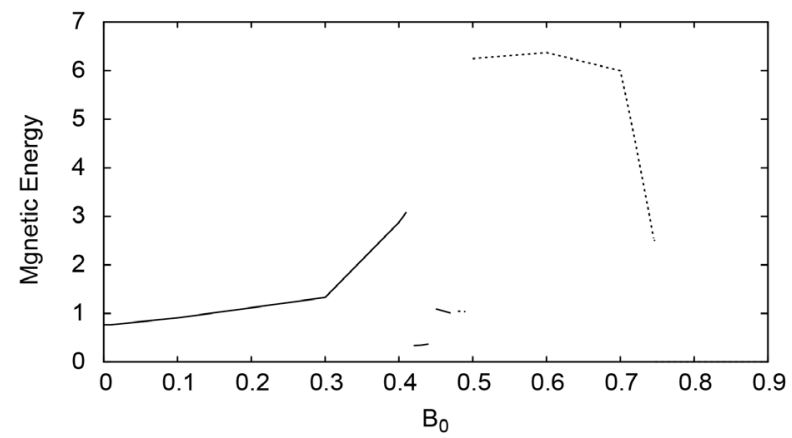

Figure 3. The QO magnetic field (solid line) and the SQ (dashid line) non-axisymmetric solutions for the system with $\boldsymbol{B}_{0} \neq 0, \boldsymbol{b}_{1}=0$.

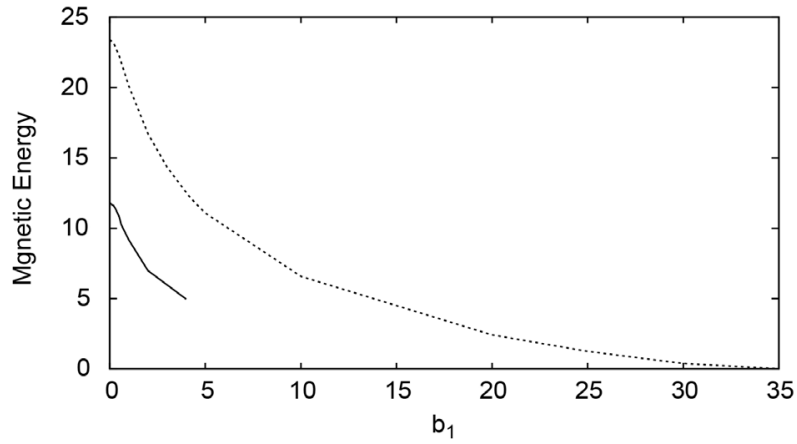

Figure 4. The OD magnetic energy (soled line) and the SD (dashed line) axisymmetric solutions for $\boldsymbol{B}_{0}=0, \boldsymbol{b}_{1} \neq 0$.

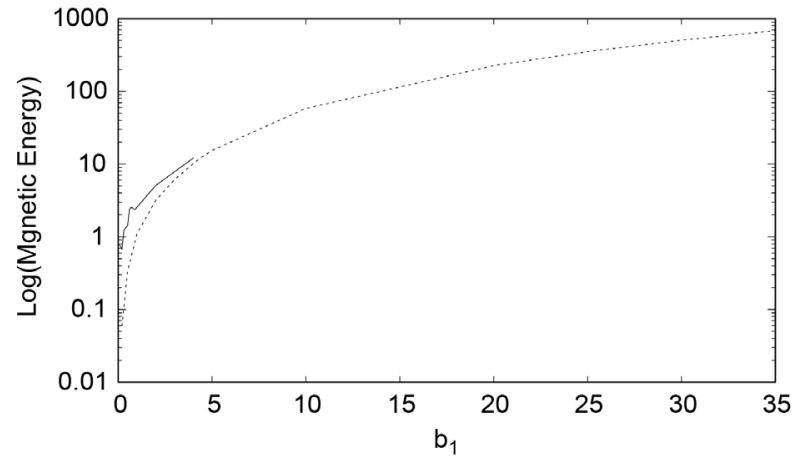

Figure 5. The OQ magnetic energy (soled line) and SQ (dashed line) non-axisymmetric solutions for $\boldsymbol{B}_{0}=0, \boldsymbol{b}_{1} \neq 0$. 


\section{Conclusion}

What is the result of imposing the external field? Both axisymmetric and nonaxisymmetric solutions are optimized by the external field, which leads to an increase of the same type of the external field and decay of the other, as when the external field is axisymmetric, this enhances the axisymmetric field generated and the disappearance of the non-axisymmetric field, as well as for the non-axisymmetric field. The behavior produced by the influence of the external field differs from what it would have been without the external field, therefore, the external field is responsible consequently; 1- The field increases with the increase in the value of the external field of influence; 2 - Some instability occurs before the transition from one behavior to another; 3 - The solutions become stable-stationary for the high value of the external field. It is very important to note that it has changed from oscillating to stationary behavior for both types.

\section{Conflicts of Interest}

The authors declare no conflicts of interest.

\section{References}

[1] Darah, A.A. and Sarson, G.R. (2007) Axisymmetric Field Generation within an Ambient Axial Field. Astronomische Nachrichten, 328, 25-35. https://doi.org/10.1002/asna.200610663

[2] Ferguson, B.B., Cain, J.C., Crider, D.H., Brain, D.A. and Harnett, E.M. (2005) External Fields on the Nightside of Mars at Mars Global Surveyor Mapping Altitudes. Geophysical Research Letters, 32, L16105. https://doi.org/10.1029/2004GL021964

[3] Crider, D.H., Vignes, D., Krymskii, A.M., Breus, T.K., Ness, N.F., Mitchell, D.L., Slavin, J.A. and Acuna, M.H. (2003) A Proxy for Determining Solar Wind Dynamic Pressure at Mars Using Mars Global Surveyor Data. Journal of Geophysical Research, 108, 1461.

[4] Acuña, M.H., Connerney, J.E.P., Wasilewski, P., Lin, R.P., Mitchell, D., Anderson, K.A., Carlson, C.W., McFadden, J., Rème, H., Mazelle, C., Vignes, D., Bauer, S.J., Cloutier, P. and Ness, N.F. (2001) Magnetic Field of Mars: Summary of Results from the Aerobraking and Mapping Orbits. Journal of Geophysical Research: Planets, 106, 23403 23417.

[5] Brain, D.A., Bagenal, F., Acuña, M.H. and Connerney J.E.P. (2003) Martian Magnetic Morphology: Contributions from the Solar Wind and Crust. Journal of Geophysical Research: Space Physics, 108, 1424. https://doi.org/10.1029/2002JA009482

[6] Qamili, E., De Santis, A., Isac, A., Mandea, M., Duka, B. and Simonyan, A. (2013) Geomagnetic Jerks as Chaotic Fluctuations of the Earth's Magnetic Field. Geochemistry, Geophysics, Geosystems, 14, 839-850.

https://doi.org/10.1029/2012GC004398

[7] Occhipinti, A., De Santis, A., and Maffei, M.E. (2014) Magnetoreception: An Unavoidable Step for Plant Evolution? Trends in Plant Science, 19, 1-4. https://doi.org/10.1016/j.tplants.2013.10.007

[8] Gómez-Péreza, N. and Wicht, J. (2010) Behavior of Planetary Dynamos under the Influence of External Magnetic Fields: Application to Mercury and Ganymede. Icarus, 209, 53-62. https://doi.org/10.1016/j.icarus.2010.04.006 
[9] Glassmeier, K.-H. (1997) The Hermean Magnetosphere and Its Ionosphere-Magnetosphere Coupling. Planetary and Space Science, 45, 119-125.

https://doi.org/10.1016/S0032-0633(96)00095-5

[10] Brian, J.A., Mario, H.A., Haje, K., James, A.S., Hideharu, U., Catherine, L.J., Michael, E.P., Sean, C.S., Jim, M.R., Thomas, H.Z., Georg, G. and Ralph, L.M. (2010) The Magnetic Field of Mercury. Space Science Reviews, 152, 307-339.

[11] Stevenson, D.J. (2000) Europa's Ocean: The Case Strengthens. Science, 289, 1305-1307. https://doi.org/10.1126/science.289.5483.1305

[12] Barenghi, C.F. (1993) Nonlinear Planetary Dynamos in a Rotating Spherical Shell. III. $\alpha^{2} \omega$ Models and the Geodynamo. Geophysical \& Astrophysical Fluid Dynamics, 71, 163-185. https://doi.org/10.1080/03091929308203601

[13] Hollerbach, R. and Jones, C.A. (1993) Influence of the Earth's Inner Core on Geomagnetic Fluctuations and Reversals. Nature, 365, 541-543. https://doi.org/10.1038/365541a0

[14] Brandenburg, A., Tuominen, I. and Radler, K.-H. (1989) On the Generation of NonAxisymmetric Magnetic Fields in Mean-Field Dynamos. Geophysical \& Astrophysical Fluid Dynamics, 49, 45-55. https://doi.org/10.1080/03091928908243462 